\title{
AUDITORIA ENERGÉTICA EM UMA AGROINDÚSTRIA DE LATICÍNIOS
}

\section{GILSON DEBASTIANI ${ }^{1}$, CARLOS E. C. NOGUEIRA ${ }^{2}$, JOSÉ H. LAWDER ${ }^{3}$, MAGNO L.VIDOTTO ${ }^{4}$, RICARDO L. AZEVEDO ${ }^{5}$}

\begin{abstract}
RESUMO: O foco deste trabalho consiste num estudo de caso referente a uma Auditoria Energética efetuada numa agroindústria de laticínios localizada na cidade de Guaíra, Extremo-Oeste paranaense. Neste contexto, o presente artigo teve por objetivos: efetuar uma auditoria energética de suas instalações, analisando o consumo de energia elétrica associado aos processos produtivos, sistemas e equipamentos existentes; identificar e quantificar as cargas instaladas na agroindústria, verificando a energia consumida e a potência demandada em cada uma delas; determinar o potencial de conservação de energia para os usos finais existentes na empresa, por meio da identificação de oportunidades para implantação de medidas que otimizassem o uso da energia; e efetuar um estudo de viabilidade econômica que apontasse para a implementação das medidas de eficiência energética. Após a análise das demandas energéticas e dos equipamentos instalados na agroindústria, destaca-se a possibilidade do aumento na oferta de energia elétrica apenas reduzindo-se o desperdício no setor industrial. A análise econômica aponta um payback descontado de 30 meses e Taxa Interna de Retorno de 3,93\% ao mês. Com a implantação das intervenções propostas, obtém-se uma redução de 11.490,50 kWh mês ${ }^{-1}$ (economia mensal de 7,26\%).
\end{abstract}

PALAVRAS-CHAVE: eficiência energética, energia elétrica, auditoria energética, redimensionamento energético.

\section{ENERGY AUDITING IN A DAIRY AGROINDUSTRIES}

ABSTRACT: The focus of this research is a case study concerning an Energy Auditing that was conducted in a dairy agribusiness located in the city of Guaira, in the extreme west of the state of Paraná. In this context, this research aimed to: perform an energy auditing of its facilities by analyzing the energy consumption associated with the processes, systems, and existing equipment, to identify and quantify the loads installed in agribusiness, energy consumed and checking the power demanded in each of them and determine the potential for energy conservation for the existing final uses at the company, by identifying opportunities to implement measures thus optimizing the use of energy, and perform an economic feasibility study that aims to implement the energy efficiency measures. After analyzing the energy demands and the equipment installed in the agribusiness, it is highlighted the possibilities of increasing the supply of electricity only reducing waste in the manufacturing sector. The economic analysis indicates a payback of 30 months and Internal Rate of Return of $3.93 \%$ per month. With the implementation of the proposed interventions, it is obtained a reduction of $11,490.50 \mathrm{kWh} \mathrm{month}^{-1}$ (monthly savings of $7.26 \%$ ).

KEYWORDS: energy efficiency, electric power, energy audit, energy resizing.

\section{INTRODUÇÃO}

A produção de leite no Brasil destaca-se como uma das principais atividades agropecuárias em razão de sua capacidade de geração de emprego e renda, e conexão com outros setores agroindustriais (FERREIRA et al., 2008). A atividade funciona como âncora na formação de renda

\footnotetext{
${ }^{1}$ Físico, Prof. Mestre, Programa de Pós-Graduação em Energia na Agricultura, UNIOESTE/Cascavel - PR, Fone: (45) 3220-7422, gilson@fag.edu.br

${ }^{2}$ Eng ${ }^{-}$Agrícola e Eletricista, Prof. Doutor, Programa de Pós-Graduação em Energia na Agricultura, UNIOESTE/Cascavel - PR

${ }^{3}$ Eng ${ }^{0}$ Eletricista, Prof. Mestre, Programa de Pós-Graduação em Energia na Agricultura, UNIOESTE/Cascavel - PR

${ }^{4}$ Eng ${ }^{0}$ Agrícola, Prof. Mestre, Programa de Pós-Graduação em Energia na Agricultura, UNIOESTE/Cascavel - PR

${ }^{5}$ Eng ${ }^{0}$ Civil, Prof. Doutor, Programa de Pós-Graduação em Energia na Agricultura, UNIOESTE/Cascavel - PR

Recebido pelo Conselho Editorial em: 16-01-2013
}

Aprovado pelo Conselho Editorial em: 28-10-2013 
e sustentação da agricultura familiar, por atuar como uma atividade central no sistema produtivo (ALTAFIN et al., 2011).

Eficiência energética é um conceito dinâmico que está associado ao crescimento econômico, à produtividade, à proteção do meio ambiente e ao desenvolvimento sustentável. É a maximização do efeito útil desejado e minimização das perdas ou irreversibilidades. Está relacionado a alguns usos finais da energia, dos quais os sistemas de ar comprimido, os sistemas frigoríficos, os motores elétricos e a iluminação são setores que acentuam as possibilidades de ganho de eficiência. Para PERRONE (2010), do ponto de vista qualitativo, há a incorporação da questão da eficiência energética não somente em relação à diminuição da conta de energia elétrica pela indústria, mas também, a partir dessa avaliação, pode-se ter uma visão sistêmica.

O dimensionamento de motores com potência acima da necessária acarreta maior custo inicial, menor rendimento e menor fator de potência (OLIVEIRA FILHO et al., 2010a). Com o aumento da automatização dos processos agrícolas, a demanda de energia elétrica para o setor agropecuário tem aumentado significativamente (OLIVEIRA FILHO et al., 2010b).

A partir da crise do petróleo, análises de equipamentos e hábitos de uso de energia passaram a ser realizadas no ponto de vista de eficiência, e descobriu-se que os serviços de energia poderiam ser proporcionados com menor gasto, afetando positivamente setores econômicos, ambientais, sociais e culturais (CEMIG, 2012).

O setor industrial tem grande parte de seu desenvolvimento impulsionado pela energia elétrica (consumo de 35,9\%), de tal forma que, juntamente com os transportes (consumo de 30,1\%) consomem atualmente 66,0\% do total de eletricidade disponível no País (EPE, 2012). Para atender ao rápido crescimento do consumo de eletricidade, foram necessários vultosos investimentos, inicialmente realizados pelo poder público, na construção de usinas hidrelétricas e na ampliação dos sistemas de transmissão e distribuição de energia elétrica, buscando alavancar o setor industrial, mas sem considerar os impactos sociais e ambientais que poderiam ser causados.

Hoje, o principal argumento contrário à construção de novas hidrelétricas é o impacto provocado sobre o modo de vida da população, flora e fauna locais, pela formação de grandes lagos ou reservatórios, aumento do nível dos rios ou alterações em seu curso após o represamento. Diante disso, a eficientização energética passou a ser considerada como um dos instrumentos capazes de garantir a oferta de energia necessária nos próximos anos. Para SELVAM et al. (2011), o Brasil como um todo tem enfrentado problemas no que se refere à geração de energia elétrica para suprir a demanda.

Segundo LUCON \& GOLDEMBERG (2009), nos países em desenvolvimento, onde o consumo per capita é pequeno, somente a eficiência energética não vai resolver o problema, porque em muitos casos os serviços energéticos finais (iluminação, aquecimento, cocção etc.) são ainda insuficientes. Porém, para TOLMASQUIM et al. (2007), no ano de 2030, a eficiência energética representará $4 \%$ do total da matriz elétrica brasileira, representando uma forma virtual de geração de energia elétrica.

As organizações perceberam a oportunidade de incorporar o desenvolvimento e a melhoria da performance econômica à ecoeficiência e à responsabilidade social (ARAÚJO \& MENDONÇA, 2009). O processo de decisão de uma empresa ou agroindústria passa pela tomada de decisão com rapidez, mas sem colocá-la em situação de risco financeiro, perda de mercado, de qualidade ou de imagem frente aos clientes (MALAFAIA et al. 2008).

NAIME \& CARVALHO (2009) destacam que, pelas carências na análise global de aspectos de infraestrutura ligados à industrialização, fatores como água, energia, tratamento e lançamento de efluentes e disposição de resíduos sólidos, que são críticos em processos industriais, frequentemente são negligenciados. 
Este trabalho realizou um estudo de eficientização de energia elétrica em uma agroindústria do setor de laticínios, localizada na cidade de Guaíra - PR. O objetivo geral foi avaliar o consumo de energia elétrica associado aos processos produtivos, sistemas e equipamentos existentes, permitindo um redimensionamento do atual parque energético para um mais eficiente. Também foi realizado o cálculo da viabilidade econômica do projeto através da avaliação da Taxa Interna de Retorno (TIR).

\section{MATERIAL E MÉTODOS}

Este trabalho foi desenvolvido em 2011, em uma agroindústria do setor de laticínios, localizada no município de Guaíra, Extremo-Oeste paranaense, local de amplitude térmica bastante acentuada, variando de $0,5^{\circ} \mathrm{C}$ a $40^{\circ} \mathrm{C}$. A agroindústria processa leite para produção de queijo, requeijão, doce de leite, iogurte e bebidas lácteas, sendo sua produção estimada em 105.000 litros de leite in natura por dia.

Os dados de carga instalada, transformador, geração própria, demanda de potência e consumo de energia são apresentados abaixo:

- Carga total instalada: 563,74 kW;

- Demanda contratada atual: $450 \mathrm{~kW}$;

- Demanda média medida: 420,33 kW;

- Transformador: 750 kVA 13,8 kV/380/220V;

- Geração própria a diesel: 500 kVA 380/220V; e

- Consumo médio mensal de energia elétrica 158.192 kWh.

A primeira etapa do trabalho caracterizou-se pela realização de um completo reconhecimento dos processos produtivos da agroindústria, identificando os equipamentos que compõem a instalação. Os dados coletados foram:

a) Históricos de demanda e consumo de energia elétrica;

b) Levantamento e identificação das características nominais e de operação dos equipamentos de força motriz (motores elétricos de indução), a partir dos dados fornecidos na placa de identificação dos motores (tipo de motor elétrico, potência, tensão e corrente nominais, rendimento e fator de potência), e a partir dos valores medidos de tensão entre fases, corrente em cada fase, fator de potência e potências ativa, reativa e aparente;

c) Avaliação do sistema de iluminação utilizado, verificando a possibilidade da substituição de lâmpadas de baixo rendimento energético por outras que apresentam maior rendimento, mantendo-se o nível de iluminância desejado. Foram verificados os tipos de lâmpadas utilizadas (incandescentes, fluorescentes, mistas, etc.), a potência e a quantidade de horas de utilização.

Os equipamentos utilizados para a obtenção dos valores de tensão, corrente e potências elétricas foram os seguintes:

- Analisador Eletrônico de Energia Digital Portátil AE-200, da marca Instrutherm;

- Wattímetro digital modelo C-80, da marca Circutor; e

- Volt-amperímetro Digital modelo ET-3110, da marca Minipa Eletrônica.

As curvas de carga diárias foram obtidas calculando-se a média dos valores de demanda medidos no período de 5 a 11-12-2011 (de segunda a domingo). Elas permitem visualizar a variação da demanda de energia elétrica na agroindústria, em função da operação das cargas elétricas existentes, durante um período de 24 horas.

As curvas de carga demonstram o perfil da demanda da empresa. Segundo informação da indústria, o período de maior produção se estabelece de segunda a sexta-feira, das 6 às 17h. A partir deste horário, as cargas vão sendo desligadas de forma escalonada até a entrada do gerador, às 17h50. Durante o horário de ponta (das 18 às 21h, e das 19 às 22h, no horário de verão), a empresa 
fica alimentada eletricamente por um gerador a diesel de $500 \mathrm{kVA}$. Durante o horário de ponta a demanda é mantida pelo gerador. Nos sábados, domingos e feriados, como não existe horário de ponta, o gerador não é ligado. 2008):

As equações utilizadas para a avaliação dos motores elétricos foram as seguintes (ANEEL,

Carregamento do motor eficiente:

$\gamma=\frac{I_{\text {med }} \cdot F P_{\text {med }}}{I_{\text {nom }} \cdot F P_{\text {nom }}} \cdot 100 \%$

em que,

$\gamma$ - carregamento do motor eficiente (percentual);

$I_{\text {med }}$ - corrente elétrica medida do motor (A);

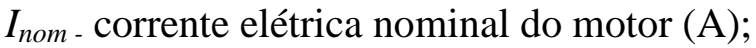

$F P_{\text {med }}$ - fator de potência medido do motor (adimensional), e

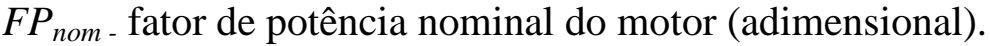

Potência ativa do motor eficiente:

$P a_{\text {mef }}=\frac{P_{\text {nom }} \cdot y}{\eta_{\text {nom }}}$

em que,

$P a_{m e f}$ - potência ativa do motor eficiente (kW);

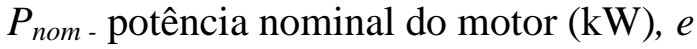

$\eta_{\text {nom - }}$ rendimento nominal do motor (percentual).

Redução de potência ativa:

$R p a=P a-P a_{m e f}$

em que,

$R p a$ - redução de potência ativa do motor $(\mathrm{kW})$, e

$\mathrm{Pa}$ - potência ativa medida do motor atual $(\mathrm{kW})$.

Redução percentual de potência ativa:

$R \%=\frac{R p a}{P a} .100 \%$

em que,

$R \%$ - redução percentual de potência ativa do motor (\%).

Energia economizada fora de ponta:

$E_{\text {efp }}=\frac{h f p \cdot R p a}{1000}$

em que,

$E_{\text {efp }}$ é a energia economizada fora do horário de ponta (MWh ano ${ }^{-1}$ ), e

hfp são as horas "fora de ponta" de funcionamento do motor por ano (h ano ${ }^{-1}$ ).

Benefício da redução de despesas:

$B r d=p_{\text {mee }} \cdot E_{\text {efp }}$

em que,

Brd é o benefício da redução de despesas $\left(\mathrm{R} \$ \mathrm{ano}^{-1}\right)$, e

$p_{\text {mee }}$ é o preço médio da energia elétrica $\left(\mathrm{R} \$ \mathrm{MWh}^{-1}\right)$. 
Foi confeccionado o plano de gestão energética industrial, que permite à empresa executar as medidas para eficientizar a utilização da energia para os usos finais analisados, de forma adequada e ordenada, permitindo visualizar a economia obtida após a adoção das medidas, ao longo de um período de tempo.

Foi realizada uma avaliação econômica das medidas de conservação de energia a partir do fluxo de caixa do investimento proposto. Foram calculados a Taxa Interna de Retorno (taxa de remuneração do capital investido que faz com que o Valor Presente Líquido seja nulo), o payback descontado (responsável por calcular o tempo de retorno ajustando os fluxos de caixa por uma taxa de juros) e o consumo específico da agroindústria (razão entre o consumo energético e a produção de leite para o período).

\section{RESULTADOS E DISCUSSÃO}

O fornecimento de energia elétrica para a agroindústria é realizado em tensão primária de distribuição $(13,8 \mathrm{kV})$. A indústria está enquadrada no subgrupo tarifário A4, incidindo tarifa horossazonal verde. Durante o horário de ponta (das 18 às 21h, e das 19 às 22h no horário de verão) a empresa é alimentada eletricamente por um gerador a diesel de $500 \mathrm{kVA}$. A Tabela 1 apresenta o histórico de consumo e demanda de energia elétrica, bem como os valores da fatura de energia elétrica.

TABELA 1. Histórico de consumo e demanda de energia elétrica em 2011. Historical consumption and electricity demand in 2011.

\begin{tabular}{|c|c|c|c|c|c|c|c|c|c|}
\hline \multirow[b]{2}{*}{ Mês } & \multicolumn{2}{|c|}{ Consumo } & \multicolumn{3}{|c|}{ Demanda } & \multirow[b]{2}{*}{$\begin{array}{c}\text {-Fator de } \\
\text { Carga }\end{array}$} & \multicolumn{2}{|c|}{ Fator de Excedente } & \multirow{2}{*}{$\begin{array}{c}\text { Fatura de } \\
\text { Energia } \\
(\mathrm{R} \$)\end{array}$} \\
\hline & $\begin{array}{l}\text { Ativo } \\
\text { (kWh) }\end{array}$ & $\begin{array}{c}\text { Reativo } \\
\text { (kVArh) }\end{array}$ & $\begin{array}{c}\text { Medida } \\
(\mathrm{kW})\end{array}$ & $\begin{array}{c}\text { Contratada } \\
(\mathrm{kW})\end{array}$ & $\begin{array}{c}\text { Faturada } \\
(\mathrm{kW})\end{array}$ & & $\begin{array}{c}\text { Potência } \\
(\%)\end{array}$ & $\begin{array}{c}\text { Reativo } \\
\text { (R\$) }\end{array}$ & \\
\hline jan. & 134.765 & 74.767 & 345 & 340 & 345 & 0,54 & 87,44 & $1.350,01$ & $29.127,73$ \\
\hline fev. & 191.131 & 70.265 & 448 & 340 & 340 & 0,58 & 93,86 & 0 & $44.448,51$ \\
\hline mar. & 132.470 & 46.694 & 415 & 340 & 340 & 0,44 & 94,31 & 0 & 29.577,39 \\
\hline abr. & 157.624 & 62.182 & 428 & 420 & 428 & 0,50 & 93,02 & 0 & $32.213,96$ \\
\hline maio & 157.551 & 45.586 & 424 & 420 & 424 & 0,51 & 96,06 & 0 & $34.305,85$ \\
\hline jun. & 138.880 & 30.614 & 404 & 420 & 420 & 0,47 & 97,68 & 0 & $30.372,16$ \\
\hline jul. & 152.558 & 34.776 & 422 & 420 & 422 & 0,50 & 97,50 & 0 & 37.372,26 \\
\hline ago. & 159.565 & 33.509 & 415 & 420 & 420 & 0,53 & 97,87 & 0 & $40.495,64$ \\
\hline set. & 157.188 & 35.851 & 419 & 420 & 420 & 0,51 & 97,50 & 0 & $40.064,05$ \\
\hline out. & 173.615 & 41.656 & 442 & 420 & 420 & 0,54 & 97,24 & 0 & $47.789,94$ \\
\hline nov. & 154.849 & 39.317 & 438 & 420 & 420 & 0,48 & 96,92 & 0 & 39.761,19 \\
\hline dez. & 188.103 & 42.754 & 444 & 420 & 420 & 0,51 & 96,84 & 0 & $39.452,37$ \\
\hline Total & 1.898 .299 & 557.971 & & & & & & $1.350,01$ & $444.981,05$ \\
\hline Média & 158.192 & 46.498 & & & & & & & $37.081,75$ \\
\hline
\end{tabular}

\section{Potencial de conservação de energia por uso final:}

a) Força Motriz: Na Tabela 2, apresenta-se uma análise econômica preliminar para a substituição dos motores existentes por motores de alto rendimento. Os cálculos utilizados para a confecção da tabela estão apresentados nas eqs. de (1) a (6). 


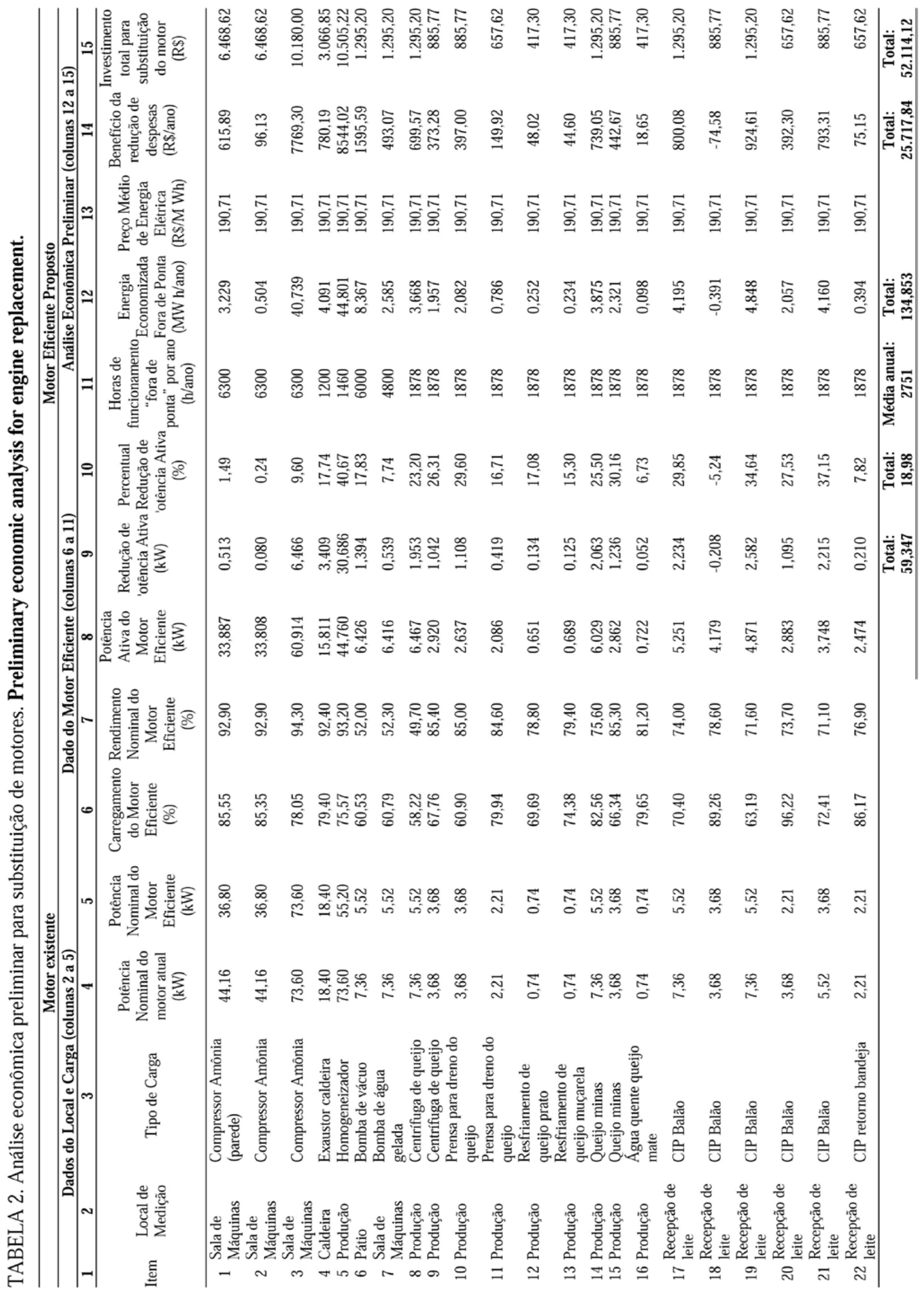


b) Iluminação: O sistema de iluminação da empresa é constituído por lâmpadas fluorescentes, incandescentes e mistas. A proposta de eficientização, organizada na Tabela 3, é substituir as lâmpadas incandescentes (LI) de $60 \mathrm{~W}$ e $100 \mathrm{~W}$ por fluorescentes compactas (LFC) de $20 \mathrm{~W}$ e 27 W, respectivamente; as lâmpadas fluorescentes tubulares (LFT) de $40 \mathrm{~W}$ por $32 \mathrm{~W}$; e as lâmpadas mistas (LM) de $160 \mathrm{~W}$ por metálicas (LMT) de $100 \mathrm{~W}$. A substituição das lâmpadas mantém os níveis de iluminância atuais e estão de acordo com a NBR 5413 - Iluminância de interiores.

TABELA 3. Proposta de eficientização do sistema de iluminação. Proposal for efficiency of the illumination system.

\begin{tabular}{|c|c|c|c|c|c|c|c|c|c|c|}
\hline \multicolumn{8}{|c|}{ Proposta de Eficientização } & \multicolumn{3}{|c|}{ Grandezas após a troca de lâmpadas } \\
\hline Local & Iluminação Atual & Lâmpada & Qtd. & $\begin{array}{c}\text { Horas } \\
\text { fora de } \\
\text { ponta }\end{array}$ & $\begin{array}{c}\text { Dias por } \\
\text { semana } \\
\text { fora de } \\
\text { ponta }\end{array}$ & $\begin{array}{c}\text { Meses de } \\
\text { funcionamento } \\
\text { durante o ano }\end{array}$ & $\begin{array}{c}\text { Potência } \\
\text { Instalada } \\
\text { (W) }\end{array}$ & $\begin{array}{l}\text { Potência } \\
\text { Máxima } \\
\text { Ativa Fora } \\
\text { de Ponta } \\
\text { (W) }\end{array}$ & $\begin{array}{c}\text { Investimento } \\
\text { (R\$) }\end{array}$ & $\begin{array}{l}\text { Consumo } \\
\text { Anual Fora } \\
\text { de Ponta } \\
\left(\mathrm{MWh}_{\text {ano }}^{-1}\right)\end{array}$ \\
\hline $\begin{array}{l}\text { Vestiário } \\
\text { Masculino }\end{array}$ & LI $60 \mathrm{~W}$ & LFC $20 \mathrm{~W}$ & 5 & 4 & 5 & 10 & 110 & 22,0 & 30,00 & 0,018 \\
\hline $\begin{array}{l}\text { Vestiário } \\
\text { Feminino }\end{array}$ & LI $60 \mathrm{~W}$ & LFC $20 \mathrm{~W}$ & 6 & 8 & 7 & 10 & 132 & 22,0 & 36,00 & 0,049 \\
\hline Banheiros & LI $100 \mathrm{~W}$ & LFC $27 \mathrm{~W}$ & 7 & 4 & 6 & 12 & 189 & 27,0 & 70,00 & 0,032 \\
\hline Produção & LFT 2 x $40 \mathrm{~W}$ & LFT $2 \times 32 \mathrm{~W}$ & 20 & 10 & 6 & 12 & 1.440 & 72,0 & 600,00 & 0,207 \\
\hline Produção & LFT $2 \times 40 \mathrm{~W}$ & LFT 2 × $32 \mathrm{~W}$ & 19 & 10 & 6 & 12 & 1.368 & 72,0 & 570,00 & 0,207 \\
\hline Produção & LFT $2 \times 40 \mathrm{~W}$ & LFT $2 \times 32 \mathrm{~W}$ & 26 & 10 & 6 & 12 & 1.872 & 72,0 & 780,00 & 0,207 \\
\hline Produção & LFT $2 \times 40 \mathrm{~W}$ & LFT 2 × $32 \mathrm{~W}$ & 24 & 10 & 6 & 12 & 1.728 & 72,0 & 720,00 & 0,207 \\
\hline Escritório & LFT $2 \times 40 \mathrm{~W}$ & LFT $2 \times 32 \mathrm{~W}$ & 10 & 10 & 6 & 12 & 720 & 72,0 & 300,00 & 0,207 \\
\hline Escritório & LFT 2 x $40 \mathrm{~W}$ & LFT 2 x $32 \mathrm{~W}$ & 10 & 10 & 6 & 12 & 720 & 72,0 & 300,00 & 0,207 \\
\hline $\begin{array}{l}\text { Sala de } \\
\text { Máquinas }\end{array}$ & LM $160 \mathrm{~W}$ & LMT $100 \mathrm{~W}$ & 6 & 13 & 7 & 12 & 660 & 110,0 & 120,00 & 0,481 \\
\hline Total & & & & & & & & & $3.525,00$ & 1,822 \\
\hline
\end{tabular}

A substituição das lâmpadas permitirá uma redução de 0,184 MWh ano-1 (de 2,006 MWh ano $^{-1}$ para 1,822 $\mathrm{MWh} \mathrm{ano}^{-1}$ ) no consumo de energia elétrica. Considerando que a tarifa de consumo fora de ponta horossazonal verde está fixada em R \$ 190,71 $\mathrm{MWh}^{-1}$, haverá uma economia anual de $\mathrm{R} \$ 35,10$ com a substituição das lâmpadas. O valor do investimento necessário para a implementação desta medida será de R \$ 3.525,00 conforme valores de material elétrico e mão de obra, pesquisados no comércio da cidade de Toledo (PR).

\section{Avaliação econômica}

A implementação de todas as medidas identificadas exigirá um investimento de R\$ 55.411,97 (valor total referente à substituição de todos os motores e lâmpadas de acordo com as Tabelas 2 e 3 , respectivamente) e proporcionará uma redução de $11.490,50 \mathrm{kWh}$ mês $^{-1}$ no consumo de eletricidade, o que significa um custo de $\mathrm{R} \$ 4,82 \mathrm{kWh}^{-1}$ para a implementação das medidas de conservação de energia. A energia economizada representaria 7,26\% (R\$ 2.191,35) do valor médio mensal da eletricidade consumida pela empresa entre os meses de janeiro e dezembro de 2011. O fluxo de caixa da Figura 1 representa, graficamente, o valor do investimento inicial para a implementação das medidas de eficiência energética e a redução mensal do custo da energia elétrica fora da ponta no decorrer do tempo. 


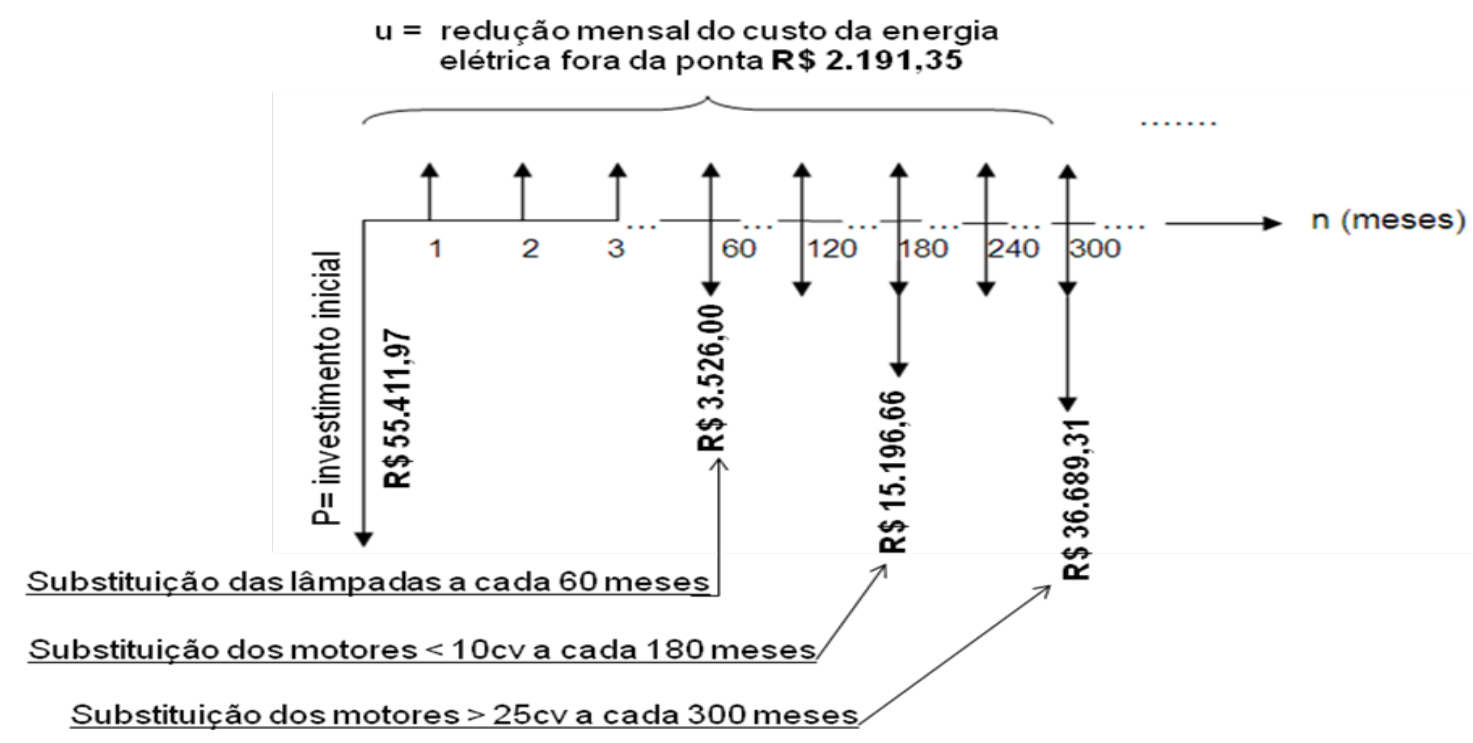

FIGURA 1. Fluxo de caixa. Cash Flow.

O payback descontado obtido para o investimento foi de 30 meses, o que demonstra que o investimento inicial se pagará em menos de 3 anos. A taxa interna de retorno obtida foi de 3,93\% a.m., estando acima da taxa mínima de atratividade considerada (1\% a.m. - taxa de juros mínima considerada para o capital investido que o torna atrativo). Já a relação custo/benefício encontrada foi de 0,30, valor menor que o estipulado pela ANEEL (2008) para projetos de eficiência energética em eletricidade. Desse modo, a substituição dos motores e das lâmpadas mostra-se uma medida viável para a eficiência energética.

\section{Consumo específico da agroindústria}

O consumo anual total de leite, em 2011, foi de 24.840.000 litros. O consumo de energia elétrica ativa no mesmo período foi de $1.898 .299 \mathrm{kWh}$. Desta relação, obtém-se o consumo específico médio (CE1) da indústria antes da implementação das medidas de eficiência energética: CE1 = $0,076421 \mathrm{kWh}$ litro $^{-1}$.

Considerando-se a implementação das medidas de eficiência energética, o consumo anual de energia elétrica passará a ser (conforme dados obtidos nas Tabelas 2 e 3): 1.898.299 - 137.886= 1.760.413 kWh. Assim, mantendo-se o mesmo processamento de leite anual, o consumo específico será: CE2 $=0,07087$ kWh litro ${ }^{-1}$.

\section{CONCLUSÕES}

Analisando-se os resultados obtidos e as condições de operação da agroindústria, propõe-se a imediata substituição dos motores elétricos atualmente utilizados por motores de alto rendimento, bem como a substituição do atual sistema de iluminação por um sistema energeticamente mais eficiente, conforme explicitado no trabalho. A redução nos gastos mensais com o consumo da energia elétrica (redução de 7,26\% a.m) evidencia o retorno financeiro do investimento em menos de três anos.

\section{REFERÊNCIAS}

ALTAFIN, I.; PINHEIRO, M. E. F.; VALONE, G. V.; GREGOLIN, A. C. Produção familiar de leite no Brasil: um estudo sobre os assentamentos de reforma agrária no município de Unai (MG). Revista UNI, Imperatriz, v.1, n.1, 2011. Disponível em: <http://www.unisulma.edu.br/Revista_UNI_artigo2_p31_49.pdf> Acesso em: 22 set 2013. 
ANEEL. Agência Nacional de Energia Elétrica. Manual para elaboração do programa de eficiência energética. Brasília: ANEEL, 2008. 63 p.

ARAÚJO, G. C.; MENDONÇA, P. S. M. Análise do processo de implantação das normas de sustentabilidade empresarial: um estudo de caso em uma agroindústria frigorifica de bovinos. Revista de Administração Mackenzi, São Paulo, v.10, n.2, 2009. Disponível em: $<$ http://www.scielo.br/scielo.php?pid=S1678-69712009000200003\&script=sci_arttext> Acesso: 25 jun. 2013.

CEMIG. COMPANHIA ENERGÉTICA DE MINAS GERAIS. Alternativas energéticas: uma visão Cemig. Belo Horizonte, CEMIG, 2012. 369 p. Disponível em:

<http://cemig20/Inovacao/AlternativasEnergeticas>. Acesso em: 20 set. 2013.

EPE. Empresa de Pesquisa Energética. Balanço Energético Nacional 2012 - Ano base 2011. Rio de Janeiro, 2012. 57 p.

FERREIRA, M. A. M.; ABRANTES, L. A.; PEREZ, R. Investigação de grupos estratégicos na indústria de laticínios por meio da abordagem multivariada. Revista de Administração Mackenzi, São Paulo, v.9, n.2, 2008. Disponível em: http://editorarevistas.mackenzie.br/index.php/RAM/article/view/161>. Acesso: 25 jun. 2013.

LUCON, O.; GOLDEMBERG, J. Crise financeira, energia e sustentabilidade no Brasil. Revista Estudos Avançados da Universidade de São Paulo, São Paulo, v. 23, n. 65, 2009. Disponível em: <http://www.scielo.br/pdf/ea/v23n65/a09v2365.pdf>. Acesso: 20 set. 2013.

MALAFAIA, G. C.; AZEVEDO, D, B.; SANTOS, A. S. Modelo de negócio na agroindústria do leite no estado do Rio Grande do Sul. Revista Brasileira de Gestão de Negócios, São Paulo, v. 10, n. 29, 2008. Disponível em: < http://www.redalyc.org/articulo.oa?id=94702903>. Acesso em: 25 jun. 2013.

NAIME, R. H.; CARVALHO, S. Análise sócioambientais das agroindústrias do vale do Rio dos Sinos - RS. Revista eletrônica do curso de Geografia, Jataí, v.13, jul. - dez., 2009. Disponível em: $<$ http://revistas.jatai.ufg.br/index.php/geoambiente/article/view/965\#.UjeP-tJ6aFo>. Acesso em: 16 set. 2013.

OLIVEIRA FILHO, D.; RIBEIRO, M. R.; MANTOVANI, E. C.; SOARES, A. A.; FERNANDES, H. C. Dimensionamento de motores para bombeamento de água. Engenharia Agrícola, Jaboticabal, v.30, n.6, 2010 a. Disponível em: <http://www.scielo.br/pdf/eagri/v30n6/a03v30n6.pdf> Acesso em: 25 jun. 2013.

OLIVEIRA FILHO, D.; TEIXEIRA, C. A.; LACERDA FILHO, A. F.; MARTINS, J. H.; QUEIROZ, J. M. Rendimento de motor elétrico como parâmetro de dimensionamento de bitola de alimentador conectado diretamente ao transformador. Engenharia Agrícola, Jaboticabal, v.30, n.5, 2010 b. Disponível em: <http://www.scielo.br/scielo.php?script=sci_arttext\&pid=S010069162010000500005\&lng=en\&nrm=iso\&tlng=pt> Acesso em: 24 jun. 2013.

PERRONE, F. D. Eficiência energética traz ganhos ambientais, financeiros e sociais para a indústria brasileira. Corrente Contínua, Brasília, v. 33, n. 234, set./out., 2010. Disponível em: <http://www.eln.gov.br/opencms/export/sites/eletronorte/modulos/correnteContinua/arquivosCC/C orrente_Continua_234_internet.pdf $>$. Acesso em: 20 set. 2013.

SELVAM, P. V.; SANTIAGO, B. H. S.; BAYER, M.; QUEIROZ, W. F. Sistema integrado de geração de energia alternativa para agroindústria de fruta e peixe usando tecnologia limpa. Revista PCH Notícias \& SHP NEWS, Itajubá, n. 25, 2011. Disponível em:

<http://www.cerpch.unifei.edu.br/resumo_art.php?id=43>. Acesso em: 16 set. 2013.

TOLMASQUIM, M. T.; GUERREIRO, A.; GORINI, R. Matriz Energética Brasileira: uma prospectiva. São Paulo: CEBRAP, 2007. Disponível em: <http://www.scielo.br/scielo.php?pid=S010133002007000300003\&script=sci_arttext>. Acesso: em 12 mar. 2013. 